\title{
ARTICULACIÓN DE SECTORES Y CRECIMIENTO ECONÓMICO EN COLOMBIA: UN ENFOQUE MULTIVARIADO DE DETECCIÓN DE CLUSTERS EMPLEANDO UN MODELO DE INSUMO PRODUCTO*
}

\author{
EDGAR DAVID GAYTÁN ALFARO"* \& JUAN CARLOS RAMOS MALLARINO*** \\ UNIVERSIDAD AUTÓNOMA DE ZACATECAS (MÉXICO)-UNIVERSIDAD LA GRAN COLOMBIA
}

Recibido/ Received/ Recebido: 03/11/2015-Aceptado/ Accepted / Aprovado: 13/09/2016

\begin{abstract}
Resumen
Considerando como base de información a la Matriz Insumo Producto para la economía colombiana del año 2010, actualizada por el Departamento Administrativo Nacional de Estadística (DANE, 2013), el presente documento emprende un proceso multivariante de reducción de datos sobre la submatriz de flujos intersectoriales con el objeto de presentar los componentes principales derivados de la intensidad de comercialización intermedia. Tales componentes representan clusters, cuya definición proviene de la intensidad de los intercambios sostenidos en las actividades económicas participantes. Los resultados pretenden convertirse en un referente de la complejidad productiva de Colombia cuya aplicación se traduzca en el desarrollo de un esquema normativo de cohesión y articulación del aparato productivo como factores de crecimiento económico.

Palabras clave: Economía colombiana; Modelos insumo producto; Análisis multivariado; Clusters; Crecimiento económico.

\section{ECONOMIC GROWTH AND SECTOR'S ARTICULATION IN COLOMBIA: A MULTIVARIATE CLUSTER DETECTION APPROACH USING AN INPUT-OUTPUT MODEL}

\begin{abstract}
Considering as a basis of information the input-output matrix for the Colombian economy in 2010, updated by the National Administrative Department of Statistics (DANE 2013), this paper undertakes a multivariate process data reduction on the submatrix of intersectoral flows in order
\end{abstract}

\footnotetext{
* El presente es un artículo de investigación. El trabajo se ha realizado en el transcurso del año 2016. Hace parte del trabajo colaborativo entre la Unidad Académica de Economía de la Universidad Autónoma de Zacatecas y la Unidad Académica de Administración de Empresas de la Universidad La Gran Colombia.

" Doctor en Ciencias Económicas de la Universidad Autónoma de Baja California, sede Tijuana. Profesor-investigador de la Unidad Académica de Economía-Universidad Autónoma de Zacatecas. Pertenece al Cuerpo Académico de Teoría Económica, UAZCA-111 y al Sistema Nacional de Investigadores del CONACYT, Nivel 1. Dirección postal: Av. Preparatoria S.N. Colonia Hidráulica, Zacatecas, Zacatecas, México. C.P. 98600. Teléfono: +52 4921336709. Correo electrónico: davidgaytan81@gmail.com

*** Doctorante en Ciencias de Gestión, Universidad Paris 13, Sorbonne Paris Cité, CEPN, A2ID, MBA Universidad Sergio Arboleda. Profesor-investigador de la Unidad Académica de Administración de Empresas-Universidad La Gran Colombia. Coordinador de la Línea de Investigación en Negocios Internacionales y Comercio Justo, indexada en Colciencias. Correo electrónico: jcrmallarino@ gmail.com
} 
to present the main components derived from the intensity of intermediate marketing. Such components represent clusters, the definition comes from the intensity of the constant exchanges in the participating economic activities. The results are intended to become a Colombia's productive complexity benchmark whose implementation will result in the development of the productive apparatus'articulation and cohesion regulatory scheme as economic growth factors.

Keywords: Colombian economy; Input-output models; Multivariate analysis; Clusters; Economic growth.

\title{
ARTICULAÇÃO DE SETORES E CRESCIMENTO ECONÔMICO NA COLÔMBIA: UM ENFOQUE MULTIVARIADO DE DETECÇÃO DE CLUSTERS EMPREGANDO UM MODELO DE INSUMO PRODUTO
}

\begin{abstract}
Resumo
Considerando como base de informação a Matriz Insumo Produto para a economia colombiana do ano 2010, atualizada pelo Departamento Administrativo Nacional de Estatística (DANE, 2013), o presente documento empreende um processo multivariado de redução de dados sobre a submatriz de fluxos intersetoriais com o objeto de apresentar os componentes principais derivados da intensidade de comercialização intermediária. Tais componentes representam clusters, cuja definição provem da intensidade dos intercâmbios sustentados nas atividades econômicas participantes. Os resultados pretendem se tornar em um referente da complexidade produtiva da Colômbia cujo aplicativo se traduza no desenvolvimento de um esquema normativo de coesão e articulação do aparelho produtivo como fatores de crescimento econômico.

Palavras chave: Economia colombiana; Modelos insumo produto; Análise multivariada; Clusters; Crescimento econômico.

Gaytán, E. \& Ramos, J. (2017). Articulación de sectores y crecimiento económico en Colombia: un enfoque multivariado de detección de clusters empleando un Modelo de Insumo Producto. En: Revista de la Facultad de Ciencias Económica: Investigación y Reflexión. rev. fac.cienc.econ, XXV (1). DOI: http://dx.doi.org/10.18359/rfce.1416
\end{abstract}

JEL: C67, C3, R12, O41.

\section{Introducción}

La amplitud y alcance de las redes de intercambio que configuran un mercado es una representación $y$, al mismo tiempo, un principio causal de la dinámica de crecimiento económico. El mercado como expresión de la concurrencia de fuerzas de oferta y demanda constituye, en términos agregados, la suma de intercambios inducidos por requerimientos para los procesos de producción y consumo final. La reiteración de la dinámica descrita, aunada a los esquemas de competencia conforman un tejido industrial cuya celeridad, denotada por cualidades de innovación, se traduce, en última instancia, en el signo inequívoco de prosperidad en una sociedad.

En el presente documento, la evaluación de las redes de intercambio para Colombia se logra a partir del tratamiento de los datos contenidos en la MIP nacional publicada por el DANE en el año 2013, con 
estadísticas para la economía colombiana de 2010. De dicha estructura de información se seleccionó, particularmente, la submatriz de demanda intermedia cuyo contenido refleja el conjunto de intercambios de bienes y servicios a una escala de desagregación de 61 subsectores. Dado lo anterior, el modelo de reducción de datos a partir del cual se evalúa la articulación de los subsectores de actividad económica partió de un total de 3,721 transacciones contenidas en la matriz de flujos intermedios.

El documento se estructura en tres secciones básicas. La primera aborda la fundamentación teórica del estudio llevando a cabo una reflexión en torno a las definiciones de cluster y agrupamiento industrial. En la misma sección se aduce la utilidad práctica del modelo insumo producto como medio para la sistematización e identificación de los intercambios económicos más relevantes en la economía colombiana como cimiente y referente en la conformación de clusters.

Una segunda sección aborda la exposición metodológica del estudio, misma que encuentra su principal referente en el trabajo de Feser \& Bergman (2000) y en la técnica multivariante de factores y componentes principales. El objetivo de dicho modelo es reducir y clasificar datos, empleando como referente económico el tamaño y variedad de los intercambios comerciales.

La sección tercera presenta los resultados haciendo uso de esquemas tabulares que distinguen los subsectores de cada agrupamiento identificado así como el tipo de relación que los primeros guardan con los segundos. Asimismo, cada tabulador es la representación lógico difusa de la pertenencia simultánea y, con diferente intensidad, del conjunto de los subsectores al extenso de los clusters detectados.

Finalmente, se presentan las conclusiones destacando la valoración normativa del estudio y la intención de que éste redunde en un referente para el diseño de una estrategia de clusters que, empleando la premisa contenida en el presente estudio (de integración multisectorial), trascienda al factor de la innovación como elemento de cohesión y funcionalidad en los mismos.

\section{Cluster y agrupamiento industrial: una precisión conceptual}

De acuerdo con Porter (2000), un cluster se define como la concentración geográfica de empresas, oferentes y proveedores especializados en un ámbito económico que compite pero que también establece lazos de cooperación. La connotación de esta definición sugiere que un cluster existe sólo en la medida en que se presenta una integración geográfica así como un régimen de producción flexible que a la postre resulta competitivo a escala global.

En apego estricto a la noción conceptual de cluster de Porter (2000) sería, de antemano, poco probable encontrar en los departamentos colombianos a un conjunto de unidades productivas que operaran bajo esa dinámica, incluso, en el país son escasas las industrias que se pueden reconocer, propiamente, como clusters.

Por lo anterior, la definición de cluster utilizada en este trabajo emana de las bases teóricas proveídas por el modelo insumo producto, es decir, se trata de un conjunto de (sub)sectores que usan cantidades relativamente grandes de los productos de los demás (sub)sectores (Laguna 2002, p. 32). Esto constituye una definición aproximada pero facilita el procedimiento técnico para la identificación de concentraciones productivas dadas por la intensidad de sus intercambios de bienes y servicios.

Siguiendo el análisis de Laguna (2002), otros aspectos a considerar en la identificación de clusters son la escala y el tipo de relaciones a partir de las cuales se edificarán. En cuanto a la escala, el proceso de identificación concibe los siguientes tipos de clusters:

- Micro clusters, que sólo contemplan empresas y unidades productivas simples;

- Meso y macro clusters, que hacen referencia a encadenamientos existentes entre clases, ramas o subsectores de actividad económica.

En cuanto al tipo de relaciones, los agrupamientos se dividen en: 
- Clusters de innovación, categoría que se ajusta con mayor precisión a la brindada por Porter (2000) y en la que se vinculan firmas o sectores que cooperan en los procesos de difusión o conocimientos nuevos, tales como aplicaciones tecnológicas o nuevos productos;

- Clusters basados en relaciones de producción, que aglutinan a empresas o sectores que participan dentro de una misma cadena de producción.

Hecha la anterior tipología, el presente documento presenta una identificación de clusters a nivel macro para la economía de Colombia al trabajar con la desagregación de 61 subsectores. De esta manera, los agrupamientos denotan relaciones de producción al ubicarse dentro de una misma cadena de intercambio de insumos incluso cuando trasciendan la delimitación geográfica del estado, propiamente dicho.

En cuanto a los clusters de innovación, éstos se consideran sólo en la medida en que a partir de la identificación de clusters basados en relaciones de producción se pueda propiciar la integración geográfica de firmas que dé pie a la difusión cruzada de ideas y, por ende, a la edificación de auténticos clusters innovadores. En otras palabras, los clusters de innovación son a este nivel de estudio y de circunstancias económicas en el estado, sólo un objetivo de política económica y una referencia conceptual que brinda orientación respecto a cómo inducir la conformación de clusters cabalmente competitivos.

La justificación de trabajar con macro clusters (por la desagregación a 61 subsectores) está dada por la posibilidad de formular políticas que fortalezcan la cohesión de todos los agentes económicos participantes en un cluster y, de esta manera, no sólo beneficiar a firmas en específico que sería una de las implicaciones del análisis de micro clusters.

Por otra parte, trabajar a nivel de micro clusters implica seguir una metodología de investigación con fuentes primarias, es decir, requiere la aplicación de encuestas o entrevistas que permitan dar cuenta de la dinámica de compras y ventas de insumos entre las diversas unidades productivas. Ello puede representar una mayor aproximación a la realidad pero también demanda más tiempo y recursos.

En virtud de lo anterior, el presente análisis parte de una base de datos ya existente: la matriz insumo producto para Colombia de 2010 de la cual se identifican clusters en virtud de la intensidad de sus vínculos productivos.

\subsection{Aplicación del modelo insumo producto}

Aplicar el modelo insumo producto en la identificación de clusters equivale a afirmar que se encontrarán los patrones de compra-venta más representativos de la tabla MIP-COLOMBIA 2010. En términos análogos, bajo esta metodología, un cluster es, al mismo tiempo, una cadena productiva cuya intensidad de relaciones económicas permite la edificación de agrupamientos intrasectoriales.

Dado que la técnica aplicada identifica cadenas productivas, no todas ellas pueden ser consideradas como clusters (en su concepto teórico puro), sin embargo, en este trabajo reciben un tratamiento como tal debido a su capacidad potencial para convertirse cabalmente en uno de ellos.

Un ejemplo paradigmático de la aplicación del modelo insumo producto en la identificación de clusters se encuentra en Feser \& Bergman (2000) quienes sugieren una serie de etapas previas a la utilización de la técnica propiamente dicha (matemática, estadística o intuitiva) que permita la conformación de los agrupamientos productivos: i) especificar el concepto de cluster ${ }^{1}$; ii) obtener los datos ${ }^{2}$; iii) identificar el método analítico ${ }^{3}$; y iv) aplicar e identificar el análisis.

1 En este trabajo, la definición de la categoría cluster parte de la idea de considerar subsectores, por lo que se trata de mesocluster y todos desde el punto de vista de la intensidad en sus relaciones intersectoriales por lo que también se hace referencia a clusters de producción.

2 La fuente directa para la conformación de clusters para Colombia es la MIP-COLOMBIA2010 proveída por el Departamento Administrativo Nacional de Estadística (DANE) de Colombia.

3 Se aplicó el análisis de factores y componentes principales. 
Los agrupamientos económicos, bajo este enfoque, se identifican a partir de la información encontrada en los eslabonamientos intersectoriales a nivel nacional y una vez hecha la regionalización de una matriz insumo producto, se detectan los encadenamientos a partir de los cuales se determinan los clusters.

Sin embargo, partir de información agregada a nivel nacional constituye una limitante ya que ello implica que para la región las condiciones de tecnología, productividad así como los patrones de consumo intermedio son iguales a los del país en su conjunto (Rey \& Mattheis 2000).

Las ventajas, no obstante, de aplicar el enfoque insumo producto, superan a sus limitantes y da la posibilidad de aplicar la técnica de factores y componentes principales cuyo detalle metodológico se explica a continuación.

\section{Metodología}

Para la elección de la técnica a partir de la cual se identificaron las redes de intercambio de Colombia se consideraron los siguientes aspectos (Dávila, 2005 , p.9): i) su viabilidad fáctica; ii) la capacidad para arrojar resultados a la brevedad; iii) el costo de su aplicación; iv) la posibilidad de trabajar con estructuras de información ampliamente desagregadas (3 y 4 dígitos); y v) su flexibilidad en cuanto a asignar patrones de localización a los clusters identificados.

Evaluando las anteriores características y contrastando metodologías como el análisis gráfico o de redes $^{4}$, el análisis multivariado ${ }^{5}$ y el de factores y componentes principales se determinó que este último reúne las condiciones óptimas para su aplica-

$4 \quad$ Utiliza teoría de grafos para determinar cadenas productivas formadas por complejas redes de industrias, posteriormente, las ordena jerárquicamente en virtud de sus capacidad para generar sinergias sobre el resto de la economía.

5 Mediante esta técnica se determina una colección de variables a partir de las cuales se seleccionan industrias con características similares y se establecen patrones algorítmicos que permiten la agrupación de las diversas unidades productivas en clusters. ción dadas las características del presente trabajo.

Una de las primeras referencias al método de factores y componentes principales se encuentra en Czamansky \& Ablas (1979) quien hace un comparativo entre diferentes alternativas para la identificación de clusters concluyendo en la robustez metodológica de esta técnica y por ende en la confiabilidad de sus resultados.

El desarrollo metodológico de este punto sigue la línea de lo propuesto por Feser \& Bergman (2000). En opinión de dichos autores, el análisis de factores y componentes principales tiene considerables ventajas entre las que destacan la posibilidad de medir los efectos directos-indirectos en los diversos encadenamientos productivos; optimiza el uso de información disponible, lo que se traduce en un ahorro de tiempo y recursos; proporciona una visión holística de la economía a partir del enfoque de clusters y al incorporar patrones de localización al análisis, es posible diseñar políticas que detonen el crecimiento de las regiones.

Los datos con los que se trabaja en esta técnica son sólo los correspondientes al cuadrante de relaciones intersectoriales de una matriz insumo producto, en este caso de la MIP-COLOMBIA 2010, es decir (Dávila, 2005, p.10):

$$
\begin{aligned}
& x_{11}+x_{12}+\ldots+x_{1 n}+y_{1}=X_{1} \\
& x_{21}+x_{22}+\ldots+x_{2 n}+y_{2}=X_{2} \\
& =\quad=\quad== \\
& ==\mathrm{=}= \\
& ===\text { = } \\
& ==== \\
& x_{n 1}+x_{n 2}+\ldots+x_{n n}+y_{n}=X_{n}
\end{aligned}
$$

Donde:

$x=$ Valor monetario de las transacciones intermedias. El primer subíndice denota al subsector de origen o vendedor y el segundo al de destino o comprador;

$y=$ Valor monetario de la demanda final; $X=$ Valor monetario del producto bruto total. 
De la expresión (1) se puede desprender la asignación de la producción que es la suma por renglones de la matriz de relaciones intersectoriales, esto es:

$$
\sum_{j=1}^{n} x_{i j}+y_{i}=X_{i}
$$

Mientras que el análisis por columnas capta las compras o el valor de los insumos utilizados en el proceso de producción por cada uno de los subsectores:

$$
\sum_{i=1}^{n} x_{i j}+z_{i}=X_{j}
$$

En seguida, la suma de las compras es denotada como $\mathrm{p}_{i}^{6}$. Por su parte, las ventas se expresan por $\mathrm{s}_{\mathrm{i}}{ }^{7}$. Tanto las ventas como las compras se analizan para los subsectores $i, j$. Por lo que habrá compras-ventas del sector $i$ así como compras-ventas del sector $j$.

El primer paso del análisis de factores y componentes principales consiste en dividir cada elemento de las relaciones intersectoriales por los montos $p_{i}$, $p_{j}, s_{i}, s_{j}$ de manera sucesiva formando las matrices $A, B, C, D$. De manera que (Feser \& Bergman, 2000, p.5):

$$
\begin{aligned}
& A=x_{i j} / p_{i(i, j=1,2, \ldots, n)} \\
& B=x_{j i} / p_{j(i, j=1,2, \ldots, n)} \\
& C=x_{i j} / s_{i(i, j=1,2, \ldots, n)} \\
& D=x_{j i} / s_{j(i, j=1,2, \ldots, n)}
\end{aligned}
$$

Posteriormente se obtienen matrices de correlación entre las cuatro matrices estimadas en el paso previo $^{8}$. En términos económicos, las correlaciones se interpretan de la siguiente manera:

- Correlación $A-B$ que mide el grado de similitud entre los patrones de compra de las industrias $i, j$.

6 Se utiliza la misma notación que Feser \& Bergman (2000). $P$ se desprende de purchases, compras.

7 De sales, ventas.

8 Dada la dimensión de las matrices $A, B, C, D$, las correlaciones se obtuvieron mediante el soporte computacional de $R$ 3.3.1.
- Correlación C-D mide la similitud entre los patrones de venta de las industrias $i, j$.

- Correlación A-D mide el grado en que los patrones de compra de $i$ son similares a los patrones de venta de $j$.

- Correlación $C-B$ mide el grado en que los patrones de venta de $i$ son similares a los patrones de compra de $j$.

El siguiente paso consiste en la formación de una matriz mixta $n \times n$ (en este caso 79x79) integrada por los valores más altos de los índices de correlación de las matrices $A, B, C, D$.

Finalmente, sobre la matriz mixta se aplica el análisis de factores y componentes principales. El núcleo de esta técnica es el problema de la obtención de los vectores y valores propios de un operador vectorial que se resuelve mediante la diagonalización de una matriz cuadrada. Cuando son extraídos los factores, es posible determinar la composición de cada cluster.

El referente técnico de la aplicación de este método se encuentra en Pérez (2004, p. 155) para quien: "El aspecto más característico del análisis factorial lo constituye su capacidad de reducción de datos. Las relaciones entre las variables observadas vienen dadas por su matriz de correlaciones, de modo que, en el análisis factorial, se puede partir de una serie de coeficientes de correlación para el conjunto de variables observadas y, a continuación, estudiar si subyace algún patrón de relaciones tal que los datos puedan ser reordenados a un conjunto menor de factores que podemos considerar como variables que recogen y resumen las interrelaciones observadas en los datos".

De esta manera, los denominados factores se convierten en colecciones de variables afines entre sí, ortogonales con respecto a otros conjuntos, es decir, independientes. Los datos por consiguiente se reducen ciñendo su unión a la interdependencia entre estos, proporcionando un conocimiento en común de su estructura subyacente. 
Este procedimiento se hizo operativo mediante la denominada Rotación de Factores, siguiendo el Método Varimax 9 . La intención de rotar los factores radica en obtener resultados que sean fácilmente interpretables. En este caso específico, el propósito es que cada una de las variables originales tenga una correlación lo más próxima a 1 con uno de los factores y correlaciones próximas a 0 con el resto de los factores. Esto significa que cada factor tendrá una correlación alta con un grupo de variables y baja con el resto de las mismas. Como solución, los factores reducen la dimensionalidad de los datos y

Siguiendo a Pérez (2004, pp. 183-184), el método varimax obtiene los ejes de los factores maximizando la suma de las varianzas de las cargas factoriales al cuadrado dentro de cada factor. La simplicidad de un factor se define por la varianza de los cuadrados de sus cargas factoriales en las variables observables, esto es:

$$
S_{i}^{2}=\frac{1}{p} \sum_{j=1}^{p}\left(l_{j i}^{2}\right)^{2}-\left(\frac{1}{p} \sum_{j=1}^{p} l_{j i}^{2}\right)^{2}
$$

El método de rotación varimax pretende hallar $\mathrm{B}=\mathrm{LT}$ de modo que la suma de las simplicidades de todos los factores sea máxima, ello implica la maximización de:

$$
S^{2}=\sum_{i=1}^{k} S_{i}^{2}=\sum_{i=1}^{k}\left[\frac{1}{p} \sum_{j=1}^{p}\left(l_{j i}^{2}\right)^{2}-\left(\frac{1}{p} \sum_{j=1}^{p} l_{j i}^{2}\right)^{2}\right]
$$

El problema dado por la expresión anterior es que las variables con mayores comunalidades tienen una mayor influencia en la solución final. Para solventar este problema se efectúa la normalización de Kaiser, en la que cada carga factorial al cuadrado se divide por la comunalidad de la variable correspondiente (a esto se conoce como el método varimax normalizado). La función a maximizar ahora será:

$$
S N^{2}=\sum_{i=1}^{k}\left[\frac{1}{p} \sum_{j=1}^{p}\left(\frac{l_{j i}^{2}}{h_{j}^{2}}\right)^{2}-\left(\frac{1}{p} \sum_{j=1}^{p} \frac{l_{j i}^{2}}{h_{j}^{2}}\right)^{2}\right]
$$

En su forma definitiva, el método varimax halla la matriz B maximizando:

$$
W=p^{2} S N^{2}=p \sum_{i=1}^{k} \sum_{j=1}^{p}\left(\frac{l_{j i}^{2}}{h_{j}^{2}}\right)^{2}-\sum_{i=1}^{n}\left(\sum_{j=1}^{p} \frac{l_{j i}^{2}}{h_{2}^{2}}\right)^{2}
$$

Una propiedad importante del método varimax es que, después de apicado, queda inalterada, tanto la varianza total explicada por los factores, como la comunalidad de cada una de las variables. La nueva matriz corresponde también a factores ortogonales y tiende a simplificar la matriz factorial por columnas, siendo muy adecuada cuando el número de factores es pequeño. consiguen develar la naturaleza de las interrelaciones existentes entre las variables originales.

\section{Aplicaciones y resultados}

Los resultados obtenidos en la rotación de factores se encuentran ponderados entre 0 y 1 denotando la intensidad con la que las unidades productivas consideradas en cada columna participan del respectivo agrupamiento ${ }^{10}$, sin excluir la posibilidad de que las actividades establezcan conectividad con otros agrupamientos, sobre todo en casos en que muestren endebles asociaciones con uno solo. Esta idea es deseable desde el punto de vista de Rey \& Mattheis (2000) para quienes una estructura más plausible de la realidad es aquella en la que los clusters no resulten excluyentes entre sí, mostrando su interconexión.

De acuerdo con Feser \& Bergman (2000, p.6) los tipos de relación que mantienen las unidades productivas (en este caso subsectores) con los diferentes clusters se clasifican siguiendo los siguientes criterios:

- $\quad 0.65<$ loading $^{11}$ : Denota un tipo de relación primaria que implica un grado de asociación alto;

- $\quad 0.5<$ loading<0.65: Relación secundaria, las unidades productivas se encuentran medianamente asociadas al agrupamiento;

- $\quad 0.35<$ loading<0.5: Las actividades participan del agrupamiento con intensidad económica poco significativa. Es un tipo de relación terciaria;

- $\quad 0.35>$ loading: Implica una relación nula. Las actividades participan de manera económicamente no significativa.

10 Un valor de 1 indicaría asociación completa y cero una independencia total con respecto al agrupamiento.

11 En este caso el loading se utiliza de manera indistinta como el Coeficiente de Asociación (C.A.). 
Como es posible inferir, este sistema de clasificación permite que las unidades económicas participen de un agrupamiento de forma primaria, en tanto, al mismo tiempo, lo pueden hacer de forma secundaria o terciaria en otros agrupamientos. Derivado de ello, los resultados buscan tener apego a la realidad en la medida en que representen el funcionamiento de clusters interdependientes.

Cabe mencionar que, originalmente, la metodología utilizada en este trabajo se aplicó al sector industrial. Los sectores primario y terciario, por su naturaleza, requieren un tratamiento distinto. La inclusión de estos sectores en el proceso de identificación de agrupamientos tendría como consecuencia un sesgo del análisis estadístico, lo que llevaría a resultados inconsistentes con los planteamientos teóricos. Su incorporación debe hacerse una vez detectados los clusters industriales, utilizando la información de las interrelaciones sectoriales contenida en las tablas de insumos (Laguna, 2002, p. 52).

No obstante a reconocer que el análisis de factores y componentes principales se ha aplicado (en trabajos previos) sólo a la manufactura (a una desagregación por ramas de 4 dígitos), en este trabajo se utilizaron las relaciones intersectoriales de la MIPCOLOMBIA 2010 que, como es posible observar, contiene actividades propias del sector primario así como del terciario.

Antes de exponer los resultados del análisis factorial, es pertinente hacer las siguientes aclaraciones:

- Los clusters que a continuación se describen no implican, necesariamente, un funcionamiento como tales ${ }^{12}$, es decir, se han conformado sólo en virtud de la intensidad de las relaciones comerciales con la que participan los subsectores que los integran;

- La estructura de cluster aquí utilizada corresponde al tipo de agrupamientos basados en relaciones de producción a escala macroeconómica;

\footnotetext{
12 En la definición pura de Porter (2000).
}

- Identificar a los diversos agrupamientos (incluso al margen de la procedencia primaria, secundaria o terciara de sus subsectores) constituye un hallazgo interesante en la medida en que se puede propiciar la integración geográfica de las unidades productivas que los integran con el ánimo de lograr una interacción cruzada de ideas entre los mismos así como todas las ventajas derivadas de los clusters de innovación.

Los resultados se expresan en la matriz de componentes rotados derivada del modelo factorial de reducción de datos. Se identificaron 10 columnas correspondientes al mismo número de clusters. La referida matriz tiene tantas filas como sectores contiene la MIP-COLOMBIA 2010 y tantas columnas como agrupamientos identificados.

El primer agrupamiento identificado, dada su dimensión, se expone en la tabla 6 (ver anexos). Se trata de un cluster que deriva, especialmente, de la naturaleza en la orientación multisectorial de la MIPCOLOMBIA 2010, primordialmente orientada a los servicios. Cuenta con actividades concomitantes primordialmente relacionadas con la generación y aprovechamiento de energía, lo que resulta congruente con el desempeño general de los procesos terciarios.

Los esquemas tabulares de los agrupamientos contienen tres columnas que hacen referencia al tipo de relación de los subsectores con respecto al cluster referido en cada tabla. Así $v$. gr. la tabla 1 contiene el subsector de Otros bienes manufacturados que pertenece al Cluster 2 en un sentido primario lo mismo que al 4 , en tanto, este mismo subsector pertenece a los Clusters 1 y 3 en sentido terciario. De esta manera se verifica el principio lógico difuso presente en la metodología de factores y componentes principales ${ }^{13}$.

\footnotetext{
13 Resultados similares los encuentran Rey \& Mattheis (2000) y Feser \& Bergman (2000). Los mismos se atribuyen a la naturaleza de interconectividad de las economías representada en el esquema de demanda intermedia del modelo insumoproducto, lo cual, a su vez, es consistente con la realidad dada la dependencia múltiple del conjunto del aparato productivo en las economías modernas.
} 
La tabla 1 contiene los resultados correspondientes al segundo agrupamiento. Por la orientación económica de los subsectores contenidos, tal agrupamiento se denomina Industria metalmecánica y procesos de transformación ${ }^{14}$. Los subsectores de Maquinaria y equipo y Otra maquinaria y suministro eléctrico ocupan la posición preponderante en cuanto a grado de pertenencia (medido por el loading). El punto medular de los procesos secundarios de la economía colombiana se expresan en el presente cluster. Se destaca la conectividad de las actividades metalmecánicas con la industria de la construcción y la producción de bebidas.

La tabla 2 expone los resultados correspondientes al Cluster 3. Destacan, por su grado de pertenencia, actividades relativas a procesos químicos y petroleros para la producción de plástico y caucho. Por su orientación productiva, este agrupamiento se denomina Procesos químicos, producción de plástico y actividades conexas.

El Cluster 4 es evidencia de la orientación gastronómica de Colombia (tabla 3). Por la orientación de los subsectores en él contenidos se ha denominado Industria alimentaria, confitería y café. Denota, asimismo, la presencia de subsectores integrantes de la cadena de valor típica de esta actividad, a saber, los productos agrícolas, su procesamiento, empaquetamiento y distribución a partir de servicios relativos al suministro de comidas y bebidas. Es, por lo anterior, un cluster de representación holística de procesos económicos de agregación de valor.

Tabla 1. C/uster 2. Industria metalmecánica y procesos de transformación. Economía de Colombia, 2010

\begin{tabular}{|c|c|c|c|c|}
\hline Subsector & Primario & Secundario & Terciario & CA-Cluster 2 \\
\hline Maquinaria y equipo & 2 & & & 0,929 \\
\hline Otra maquinaria y suministro eléctrico & 2 & & & 0,921 \\
\hline $\begin{array}{l}\text { Trabajos de construcción, construcción y reparación de edificaciones y servicios de arren- } \\
\text { damiento de equipo con operario }\end{array}$ & 2 & & & 0,886 \\
\hline Productos metalúrgicos básicos (excepto maquinaria y equipo) & 2 & & & 0,881 \\
\hline $\begin{array}{l}\text { Trabajos de construcción, construcción de obras civiles y servicios de arrendamiento de } \\
\text { equipo con operario }\end{array}$ & 2 & & 1 & 0,84 \\
\hline Otros bienes manufacturados ncp & 2,4 & & 1,3 & 0,792 \\
\hline Bebidas & & 1,2 & & 0,617 \\
\hline Productos minerales no metálicos & & & $2,5,11$ & 0,452 \\
\hline Minerales no metálicos & 5 & & 1,2 & 0,399 \\
\hline Minerales metálicos & & 5 & $1,2,11$ & 0,388 \\
\hline
\end{tabular}

Fuente: Elaboración propia con datos de la MIP-COLOMBIA 2010 y los resultados del análisis de factores y componentes principales.

14 El nombre de este y los demás clusters se establece a criterio de los autores considerando el tipo de actividades económicas prevalecientes, su orientación productiva y la clase de interconexión con el resto de la complejidad económica de Colombia. 
Tabla 2. Cluster 3. Procesos químicos, producción de plástico y actividades conexas. Economía de Colombia, 2010

\begin{tabular}{|c|c|c|c|c|}
\hline Subsector & Primario & Secundario & Terciario & CA-Cluster 3 \\
\hline Productos de caucho y de plástico & 3 & & & 0,961 \\
\hline Sustancias y productos químicos & 3 & & & 0,955 \\
\hline Productos de café & 3 & & & 0,932 \\
\hline Otros productos agrícolas & 3 & & 4 & 0,896 \\
\hline Curtido y preparado de cueros, productos de cuero y calzado & 3 & 8 & & 0,669 \\
\hline Artículos textiles, excepto prendas de vestir & & $1,3,8$ & & 0,627 \\
\hline Cacao, chocolate y productos de confitería & 4 & & 3 & 0,405 \\
\hline Otros bienes manufacturados NCP & 2,4 & & 1,3 & 0,352 \\
\hline
\end{tabular}

Fuente: Elaboración propia con datos de la MIP-COLOMBIA 2010 y los resultados del análisis de factores y componentes principales.

Tabla 3. Cluster 4. Industria alimentaria, confitería y café. Economía de Colombia, 2010

\begin{tabular}{|c|c|c|c|c|}
\hline Subsector & Primario & Secundario & Terciario & CA-Cluster 4 \\
\hline Azúcar y panela & 4 & & & 0,925 \\
\hline Productos de molinería, almidones y sus productos & 4 & & & 0,915 \\
\hline Productos de tabaco & 4 & & & 0,898 \\
\hline Cacao, chocolate y productos de confitería & 4 & & 3 & 0,83 \\
\hline Aceites y grasas animales y vegetales & 4 & & & 0,817 \\
\hline Otros bienes manufacturados ncp & 2,4 & & 1,3 & 0,766 \\
\hline Servicios de alojamiento, suministro de comidas y bebidas & 4 & 7 & & 0,655 \\
\hline Fibras textiles naturales, hilazas e hilos; tejidos de fibras textiles, incluso afelpados & & & 4 & 0,464 \\
\hline Otros productos agrícolas & 3 & & 4 & 0,356 \\
\hline
\end{tabular}

Fuente: Elaboración propia con datos de la MIP-COLOMBIA 2010 y los resultados del análisis de factores y componentes principales.

En la tabla 4 se muestran los resultados correspondientes a la conformación del Cluster 5. Es notoria la presencia de subsectores característicos de la industria del transporte. Destaca, asimismo, la inclusión de la industria minera metálica y no metálica, lo que sugiere la existencia de procesos transformadores, basados en minerales, de componentes para medios de transporte. Esto es particularmente lógico dada la inclusión de hidrocarburos en el ámbito de la minería no metálica.
La tabla 5 presenta un resumen de los agrupamientos restantes identificados. El petróleo, los alimentos cárnicos, la industria textil, el aprovechamiento de celulosa y la generación de energía eléctrica constituyen cotos industriales con niveles de comercialización intermedia significativa para la definición de cluster contemplada en el presente documento.

En todos los agrupamientos y, como elemento característico de la metodología empleada, puede constatarse la inclusión de actividades económicas que, en apariencia, no guardan relación empírica 
con la definición del cluster en turno, empero, esto constituye un hallazgo relevante puesto que la valía de un agrupamiento sinérgico estriba en la extensión de redes de proveeduría incluyentes y co-participativas con sectores nominalmente inconexos. Ejemplo de lo anterior son los Servicios de alojamiento, suministro de comidas y bebidas adscritos al Cluster 7 de Alimentos cárnicos y deriva- dos. Tal resultado es sugerente de la conectividad de las actividades turísticas y hoteleras con la industria alimentaria. La correspondencia entre generación de alimentos, principalmente cárnicos, y la movilidad turística es, bajo esta lógica, un detonante de la integración multisectorial y, por ende, empresarial al identificarse las unidades económicas específicas que integran a cada subsector.

Tabla 4. Cluster 5. Industria del transporte. Economía de Colombia, 2010

\begin{tabular}{|c|c|c|c|c|}
\hline Subsector & Primario & Secundario & Terciario & CA-Cluster 5 \\
\hline Servicios de transporte terrestre & 5 & & & 0,965 \\
\hline Servicios de transporte por vía aérea & 5 & & & 0,903 \\
\hline Servicios de transporte por vía acuática & 5 & & & 0,885 \\
\hline Minerales no metálicos & 5 & & 1,2 & 0,692 \\
\hline Minerales metálicos & & 5 & $1,2,11$ & 0,549 \\
\hline Productos minerales no metálicos & & & $2,5,11$ & 0,438 \\
\hline Carbón mineral & & 11 & 1,5 & 0,427 \\
\hline
\end{tabular}

Fuente: Elaboración propia con datos de la MIP-COLOMBIA 2010 y los resultados del análisis de factores y componentes principales.

Tabla 5. Esquema resumen de agrupamientos industriales 6-10. Economía de Colombia, 2010

Tabla 5.1. Cluster 6. Industria del petróleo

\begin{tabular}{|c|c|c|c|c|}
\hline Subsector & Primario & Secundario & Terciario & CA-Cluster 6 \\
\hline Petróleo crudo, gas natural y minerales de uranio y torio & 6 & & & 0,94 \\
\hline Productos de la refinación del petróleo; combustible nuclear & 6 & & & 0,919 \\
\hline Gas domiciliario & 6 & 1 & & 0,754 \\
\hline
\end{tabular}

Tabla 5.2. Cluster 7. Alimentos cárnicos y derivados

\begin{tabular}{|c|c|c|c|c|}
\hline Subsector & Primario & Secundario & Terciario & CA-Cluster 7 \\
\hline Productos de la pesca, la acuicultura y servicios relacionados & 7 & & & 0,904 \\
\hline Animales vivos, productos animales y productos de la caza & 7 & & & 0,845 \\
\hline Servicios de alojamiento, suministro de comidas y bebidas & 4 & 7 & & 0,562 \\
\hline Carnes y pescados & 12 & & 7 & 0,491 \\
\hline
\end{tabular}


Tabla 5.3. Cluster 8. Industria textil y aprovechamiento de fibras

\begin{tabular}{|l|c|c|c|c|}
\hline \multicolumn{1}{|c|}{ Subsector } & Primario & Secundario & Terciario & CA-Cluster 8 \\
\hline Muebles & 8 & & 0,783 \\
\hline Curtido y preparado de cueros, productos de cuero y calzado & 3 & 8 & 0,531 \\
\hline Artículos textiles, excepto prendas de vestir & & $1,3,8$ & & 0,527 \\
\hline Tejidos de punto y ganchillo; prendas de vestir & 1 & & 8 & 0,481 \\
\hline
\end{tabular}

Tabla 5.4. Cluster 9. Aprovechamiento de celulosa e industria del papel

\begin{tabular}{|l|c|c|c|c|}
\hline \multicolumn{1}{|c|}{ Subsector } & Primario & Secundario & Terciario & CA-Cluster 9 \\
\hline Productos de madera, corcho, paja y materiales trenzables & 9 & & & 0,814 \\
\hline Productos de papel, cartón y sus productos & & & 1,9 & 0,462 \\
\hline
\end{tabular}

Tabla 5.5. Cluster 10. Procesos generadores de energía eléctrica

\begin{tabular}{|l|c|c|c|c|}
\hline \multicolumn{1}{|c|}{ Subsector } & Primario & Secundario & Terciario & CA-Cluster 11 \\
\hline Energía eléctrica & 11 & & & 0,777 \\
\hline Carbón mineral & & 11 & 1,5 & 0,562 \\
\hline Minerales metálicos & & 5 & $1,2,11$ & 0,495 \\
\hline Productos minerales no metálicos & & & $2,5,11$ & 0,458 \\
\hline
\end{tabular}

Fuente: Elaboración propia con datos de la MIP-COLOMBIA 2010 y los resultados del análisis de factores y componentes principales.

\section{Conclusiones}

La aplicación del enfoque de clusters es relativamente nuevo considerando el empleo de una matriz insumo-producto. El proceso técnico metodológico implicó la detección de agrupamientos y el cálculo del grado de pertenencia de los subsectores, medido con coeficientes de asociación obtenidos del análisis multivariado de factores y componentes principales para, finalmente, representarlos en tablas ponderando pertenencia y monto de valor comercializado. De esta forma, el ordenamiento de los subsectores en cada cluster no es fortuito, da una idea de la lejanía o cercanía que éstos tienen con respecto a la actividad que denota, en mayor medida, el rasgo característico de especialización. Éste procedimiento, además de arrojar el resultado concreto de detec- ción de agrupamientos, también significó un medio para verificar la consistencia de los resultados del análisis factorial. Al contrastar los agrupamientos identificados con su dinámica de intercambios específica, en todos los casos resultó evidente que los subsectores integrantes de cada agrupamiento mostraban mayores montos de valor comercializado entre ellos que con respecto al resto de la estructura productiva. En los clusters, en promedio más pequeños, fue, también, evidente el parentesco entre las actividades integrantes.

Atendiendo a los resultados y siguiendo los criterios que permiten identificar a un agrupamiento como un cluster de innovación se pude inferir que, en el caso de Colombia, los clusters se encuentran en una fase embrionaria, es decir, la integración de cadenas 
productivas sólo constituyen una aproximación respecto a la posibilidad de conformar un agrupamiento interindustrial cuyas características preeminentes sean la diseminación de conocimientos y habilidades orientados a la innovación.

El término cluster tiene implícita la noción estratégica de competitividad, la forma de capitalizar las oportunidades que brinda esta modalidad de organización industrial depende, en gran medida, de la adopción de un esquema de políticas públicas que induzca la formación de cuadros de innovación reconocidos como el hilo conductor de acción entre diversas instituciones, públicas y privadas.

Si bien, lo aquí expuesto busca, ante todo, abonar a la representación de un mapa del tejido industrial de Colombia, sus resultados, contando con el aval otorgado por el tratamiento metodológico de los datos, pretenden ser el referente que derive en la orientación de los senderos de intercambios, (dados en el contexto del quehacer de los agentes económicos y de gobierno), con el objeto de hacerlos planificados y con inclusiones subsectoriales justificadas que se traduzcan, en última instancia, en una consideración fundamentada de la estrategia de clusters y sus efectos en el crecimiento económico del país.

\section{Referencias}

Czamansky, S. \& Ablas, L. (1979). Identification of industrial clusters and complexes: a comparision of methods and findings. Urban Studies, 16.

Dávila, A. (2005). Industrial Clusters in Mexico 1988-2002. En: R. Rabellotti, E. Giuliani \& P. Meine (eds.), Clusters facing competition: the importance of external linkages, Hampshire, Ashgate, pp. 231.257.

DANE-Departamento Administrativo Nacional de Estadística (2013). Recuperado de https://www.dane.gov.co/files/investigaciones/ pib/especiales/metodologia_matriz_insumo_producto.pdf

Feser, E. \& Bergman, E. (2000). National industry cluster templates: a framework for applied regional cluster analysis, Regional Studies, 34 (1).

Laguna, C. (2002). Identificación de clusters industriales regionales en la economía del Estado de Coahuila. Tesis de Maestría en Economía Regional. Centro de Investigaciones Socioeconómicas. Universidad Autónoma de Coahuila.

Pérez, C. (2004). Técnicas de análisis multivariante de datos. Pearson Educación. Madrid, España.

Porter, M. (2000). Location, competition, and economic development: local clusters in a global economy. Economic Development Quarterly, 14 (1).

Rey, S. \& Mattheis, D. (2000). Identifying regional industrial clusters in California. Report prepared for the California Employment Development Department. San Diego State University. 


\section{ANEXOS}

Tabla 6. Cluster 1. Servicios y actividades concomitantes. Economía de Colombia, 2010

\begin{tabular}{|c|c|c|c|c|}
\hline Subsector & Primario & Secundario & Terciario & CA-Cluster 1 \\
\hline Servicios de enseñanza de mercado & 1 & & & 0,988 \\
\hline $\begin{array}{l}\text { Servicios de asociaciones y esparcimiento, culturales, deportivos y } \\
\text { otros servicios de no mercado }\end{array}$ & 1 & & & 0,979 \\
\hline Servicios a las empresas excepto servicios financieros e inmobiliarios & 1 & & & 0,979 \\
\hline Servicios de correos y telecomunicaciones & 1 & & & 0,977 \\
\hline Comercio & 1 & & & 0,967 \\
\hline $\begin{array}{l}\text { Servicios de alcantarillado y eliminación de desperdicios, saneamiento } \\
\text { y otros servicios de protección del medio ambiente }\end{array}$ & 1 & & & 0,965 \\
\hline Servicios de intermediación financiera, de seguros y servicios conexos & 1 & & & 0,963 \\
\hline Servicios complementarios y auxiliares al transporte & 1 & & & 0,956 \\
\hline $\begin{array}{l}\text { Servicios de asociaciones y esparcimiento, culturales, deportivos y } \\
\text { otros servicios de mercado }\end{array}$ & 1 & & & 0,95 \\
\hline Agua & 1 & & & 0,946 \\
\hline Servicios de enseñanza de no mercado & 1 & & & 0,889 \\
\hline Servicios inmobiliarios y de alquiler de vivienda & 1 & & & 0,886 \\
\hline Tejidos de punto y ganchillo; prendas de vestir & 1 & & 8 & 0,842 \\
\hline $\begin{array}{l}\text { Administración pública y defensa; dirección, administración y control } \\
\text { del sistema de seguridad social }\end{array}$ & 1 & & & 0,836 \\
\hline Edición, impresión y artículos análogos & 1 & & & 0,793 \\
\hline Gas domiciliario & 6 & 1 & & 0,597 \\
\hline Artículos textiles, excepto prendas de vestir & & $1,3,8$ & & 0,52 \\
\hline Bebidas & & 1,2 & & 0,516 \\
\hline Otros bienes manufacturados ncp & 2,4 & & 1,3 & 0,445 \\
\hline Minerales no metálicos & 5 & & 1,2 & 0,392 \\
\hline Minerales metálicos & & 5 & $1,2,11$ & 0,391 \\
\hline Productos de papel, cartón y sus productos & & & 1,9 & 0,386 \\
\hline $\begin{array}{l}\text { Trabajos de construcción, construcción de obras civiles y servicios de } \\
\text { arrendamiento de equipo con operario }\end{array}$ & 2 & & 1 & 0,383 \\
\hline Carbón mineral & & 11 & 1,5 & 0,362 \\
\hline
\end{tabular}

Fuente: Elaboración propia con datos de la MIP-COLOMBIA 2010 y los resultados del análisis de factores y componentes principales. 


\begin{tabular}{|c|c|c|c|c|c|c|c|c|c|c|c|c|c|c|c|c|c|c|c|c|c|c|}
\hline & & 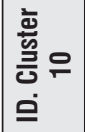 & $\begin{array}{l}\dot{0} \\
\dot{0} \\
0\end{array}$ & $\begin{array}{l}\bar{y} \\
0 \\
0\end{array}$ & $\begin{array}{l}0 \\
0 \\
0 \\
0\end{array}$ & $\tilde{\delta}$ & $\begin{array}{l}\ddot{0} \\
\vdots \\
0\end{array}$ & $\begin{array}{l}\widetilde{0} \\
i \\
0 \\
0\end{array}$ & $\begin{array}{l}\text { षे } \\
0\end{array}$ & 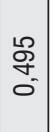 & $\begin{array}{l}\hat{m} \\
\tilde{O}\end{array}$ & $\mid \begin{array}{l}g \\
0 \\
0\end{array}$ & $\begin{array}{l}\widetilde{\Xi} \\
\tilde{\sigma}\end{array}$ & $\mid \begin{array}{l}\bar{y} \\
\overline{0} \\
0 \\
\end{array}$ & $\begin{array}{l}\mathcal{F} \\
0 \\
0\end{array}$ & 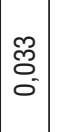 & $\begin{array}{l}\mathcal{Y} \\
\text { Oे } \\
0\end{array}$ & $\begin{array}{l}\dot{0} \\
0 \\
0\end{array}$ & $\begin{array}{l}\dot{0} \\
\dot{0} \\
0\end{array}$ & $\mid \begin{array}{c}\pi \\
\frac{\pi}{6} \\
0\end{array}$ & $\begin{array}{l}\tilde{\Xi} \\
0 \\
0\end{array}$ & ס्ర్రి \\
\hline & 禀 & 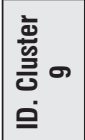 & $\begin{array}{l}\forall \\
0 \\
0 \\
0\end{array}$ & $\mid \begin{array}{l}\hat{0} \\
\hat{0} \\
0\end{array}$ & $\begin{array}{l}\infty \\
: 0 \\
0 \\
0\end{array}$ & $\begin{array}{l}\hat{o} \\
\hat{o}\end{array}$ & $\begin{array}{l}\widetilde{\tilde{O}} \\
\text { o. }\end{array}$ & $\begin{array}{l}\bar{\sigma} \\
0 \\
0\end{array}$ & $\begin{array}{l}\infty \\
\text { o̊ } \\
0\end{array}$ & $\begin{array}{l}\infty \\
0 \\
0 \\
0\end{array}$ & $\begin{array}{l}\mathbb{2} \\
\text { ర్ } \\
0\end{array}$ & $\mid \begin{array}{l}0 \\
0 \\
0 \\
0\end{array}$ & $\begin{array}{l}\hat{D} \\
0 \\
0\end{array}$ & $\bar{E}$ & $\begin{array}{l}8 \\
0 \\
0\end{array}$ & $\mid \begin{array}{l}0 \\
0 \\
0 \\
0\end{array}$ & $\begin{array}{l}0 \\
0 \\
0 \\
0\end{array}$ & $\begin{array}{l}\mathscr{0} \\
0 \\
0 \\
0\end{array}$ & $\begin{array}{c}0 \\
0 \\
0\end{array}$ & 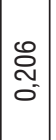 & $\begin{array}{l}\stackrel{\Xi}{\square} \\
\vdots \\
0\end{array}$ & $\begin{array}{l}\frac{0}{6} \\
\frac{10}{0}\end{array}$ \\
\hline & 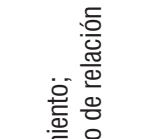 & 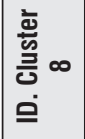 & $\begin{array}{l}\infty \\
8 \\
0\end{array}$ & 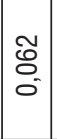 & : & $\begin{array}{l}8 \\
: \\
0\end{array}$ & $\frac{\widetilde{\sigma}}{0}$ & $\begin{array}{l}0 \\
0 \\
0 \\
0\end{array}$ & ஜ: & $\begin{array}{l}\overline{0} \\
0 \\
0\end{array}$ & $\begin{array}{l}\tilde{O} \\
0 \\
0\end{array}$ & $\mid \begin{array}{l}g \\
0 \\
0\end{array}$ & $\begin{array}{l}0 \\
0 \\
0 \\
0\end{array}$ & $\begin{array}{l}\tilde{\sigma} \\
\tilde{\sigma} \\
0\end{array}$ & 兽 & $\mid \begin{array}{c}\overline{0} \\
\overline{0}\end{array}$ & $\begin{array}{l}\tilde{O} \\
0 \\
0\end{array}$ & $\begin{array}{l}\tilde{\sigma} \\
0 \\
0\end{array}$ & $\begin{array}{l}\tilde{D} \\
0 \\
0\end{array}$ & $\mid$ & $\begin{array}{l}0 \\
0 \\
0 \\
0\end{array}$ & $\hat{0}$ \\
\hline & 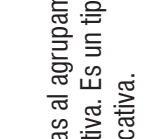 & 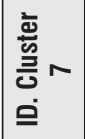 & 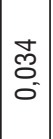 & 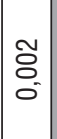 & $\begin{array}{l}\text { L8 } \\
0 \\
0 \\
0\end{array}$ & $\begin{array}{l}\text { O্ } \\
\text { O }\end{array}$ & $\begin{array}{l}\dot{\Delta} \\
\text { O্. }\end{array}$ & $\delta_{0}$ & $\begin{array}{l}\infty \\
\varnothing \\
0\end{array}$ & $\begin{array}{l}0 \\
0 \\
0 \\
0\end{array}$ & $\begin{array}{l}\bar{\sigma} \\
0 \\
\sigma^{\circ}\end{array}$ & 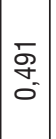 & $\begin{array}{l}\overline{8} \\
0\end{array}$ & $\begin{array}{c}\hat{\tilde{N}} \\
0 \\
0\end{array}$ & $\mid$ & $\mid \begin{array}{l}0 \\
0 \\
0\end{array}$ & $\begin{array}{l}\tilde{0} \\
0 \\
0\end{array}$ & $\frac{12}{0}$ & $\begin{array}{l}\tilde{2} \\
\tilde{0} \\
0\end{array}$ & $\begin{array}{l}0 \\
0 \\
0\end{array}$ & 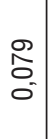 & $\frac{0}{0}$ \\
\hline & 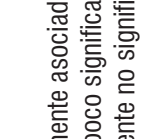 & 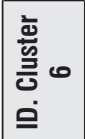 & $\begin{array}{l}8 \\
0 \\
0\end{array}$ & $\mid \begin{array}{l}0 \\
\tilde{O} \\
0 \\
0\end{array}$ & $\begin{array}{l}\text { ôे } \\
\text { : }\end{array}$ & $\begin{array}{l}\infty \\
\text { O্் } \\
0\end{array}$ & $\begin{array}{l}\stackrel{+}{\Delta} \\
0 \\
0\end{array}$ & 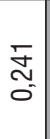 & $\begin{array}{l}\text { मे } \\
\text { ( }\end{array}$ & $\begin{array}{l}\hat{\ddot{o}} \\
0\end{array}$ & $\begin{array}{l}\hat{8} \\
0 \\
0\end{array}$ & $\mid \begin{array}{l}\overline{5} \\
\frac{5}{0}\end{array}$ & $\underset{0}{\stackrel{N}{*}}$ & $\mid \begin{array}{l}\frac{8}{9} \\
\frac{1}{0}\end{array}$ & $\begin{array}{l}1 \\
\text { ò } \\
0 \\
0\end{array}$ & $\mid \begin{array}{l}0 \\
\frac{i}{6} \\
0 \\
0\end{array}$ & $\begin{array}{l}0 \\
0 \\
0 \\
0\end{array}$ & $\begin{array}{l}\tilde{O} \\
\tilde{O} \\
0\end{array}$ & $\begin{array}{l}2 \\
0 \\
0 \\
0\end{array}$ & $\mid \begin{array}{c}\infty \\
\infty \\
0 \\
0\end{array}$ & $\begin{array}{l}0 \\
0 \\
0 \\
0\end{array}$ & क्. \\
\hline & 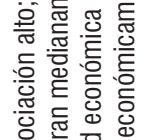 & 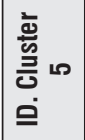 & $\mid \begin{array}{l}2 \\
0 \\
0 \\
0\end{array}$ & $\begin{array}{l}8 \\
8 \\
0 \\
0\end{array}$ & $\begin{array}{l}\text { N } \\
\stackrel{0}{0}\end{array}$ & $\begin{array}{l}8 \\
0\end{array}$ & O & $\begin{array}{l}\hat{\vartheta} \\
\text { जै } \\
0\end{array}$ & $\begin{array}{l}\text { I. } \\
\text { O. }\end{array}$ & $\begin{array}{l}\text { g } \\
0 \\
0 \\
0\end{array}$ & 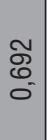 & $\mid \begin{array}{l} \pm \\
\\
0\end{array}$ & $\begin{array}{l}\infty \\
: \\
0 \\
0\end{array}$ & $\mid \begin{array}{l}0 \\
0 \\
0 \\
0\end{array}$ & $\begin{array}{l}1 \\
0 \\
0 \\
0\end{array}$ & $\mid \begin{array}{l}0 \\
0 \\
0 \\
0\end{array}$ & $\begin{array}{l}0 \\
\frac{0}{0}\end{array}$ & $\begin{array}{l}\bar{\sigma} \\
0 \\
0\end{array}$ & $\begin{array}{l}\infty \\
0 \\
0\end{array}$ & $\mid \begin{array}{l}1 \\
0 \\
0 \\
0\end{array}$ & $\begin{array}{l} \pm \\
\stackrel{\Xi}{0}\end{array}$ & $\frac{\hat{m}}{0}$ \\
\hline & 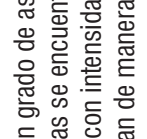 & 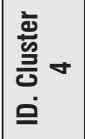 & $\begin{array}{l}0 \\
0 \\
0 \\
0\end{array}$ & $\begin{array}{l}0 \\
0 \\
m \\
0 \\
0\end{array}$ & $\begin{array}{l}\frac{\infty}{5} \\
\frac{1}{0}\end{array}$ & $\frac{\infty}{\frac{\infty}{0}}$ & $\begin{array}{l}\widetilde{O} \\
\text { Oे } \\
0\end{array}$ & $\sigma_{0}$ & $\frac{\approx}{\overline{0}}$ & $\underset{\Xi}{\cong}$ & $\frac{\mathscr{P}}{\stackrel{-}{\circ}}$ & \begin{tabular}{|l} 
L \\
م. \\
o
\end{tabular} & $\begin{array}{l}n \\
\overline{0} \\
0 \\
0\end{array}$ & $\mid$ & $\mid$ & $\mid \begin{array}{l}\infty \\
\stackrel{\infty}{+} \\
0\end{array}$ & 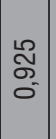 & $\begin{array}{c}\mathscr{2} \\
\infty \\
0 \\
0\end{array}$ & $\begin{array}{l}8 \\
\varnothing \\
0 \\
0\end{array}$ & 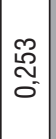 & $\begin{array}{l}\infty \\
0 \\
0 \\
0\end{array}$ & 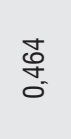 \\
\hline & 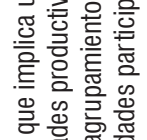 & 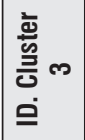 & $\begin{array}{l}\widetilde{2} \\
\tilde{\sigma} \\
0\end{array}$ & $\begin{array}{l}0 \\
8 \\
0 \\
0\end{array}$ & $\begin{array}{l}\bar{o} \\
\stackrel{0}{0}\end{array}$ & $\begin{array}{l}\text { : } \\
\text { ○ }\end{array}$ & 兽 & $\begin{array}{l}\bar{m} \\
\bar{\sigma}\end{array}$ & $\frac{0}{\frac{0}{0}}$ & $\mid \begin{array}{l}5 \\
\frac{5}{0} \\
0\end{array}$ & $\begin{array}{l}\bar{\Xi} \\
\overline{0}\end{array}$ & $\mid \begin{array}{l}\infty \\
\vdots \\
0 \\
0\end{array}$ & $\begin{array}{l}0 \\
0 \\
0\end{array}$ & $\mid \begin{array}{l}\infty \\
0 \\
0 \\
0\end{array}$ & $\mid \begin{array}{l}\mathbf{1} \\
\stackrel{0}{0} \\
0\end{array}$ & $\mid \begin{array}{l}0 \\
\frac{0}{0} \\
0\end{array}$ & $\begin{array}{l}\tilde{y} \\
0 \\
0 \\
0\end{array}$ & 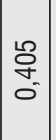 & $\begin{array}{l}\tilde{N} \\
\tilde{\omega} \\
0\end{array}$ & $\mid \begin{array}{c}\mathscr{2} \\
\stackrel{2}{0} \\
0\end{array}$ & $\begin{array}{l}\overline{0} \\
\text { o }\end{array}$ & $\begin{array}{l}\underset{N}{N} \\
\text { O }\end{array}$ \\
\hline & 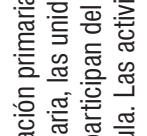 & 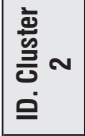 & $\begin{array}{l}\text { वे } \\
0 \\
0\end{array}$ & $\mid \begin{array}{l}0 \\
0 \\
0 \\
0\end{array}$ & $\begin{array}{l}\frac{\wp}{\circ} \\
\frac{0}{0}\end{array}$ & $\begin{array}{l}\text { 芯 } \\
\text { O. }\end{array}$ & $\begin{array}{l}\mathbb{\Xi} \\
\text { O. }\end{array}$ & $\bar{\sigma}_{0}$ & $\frac{\mathscr{2}}{0}$ & 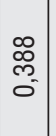 & ్ㅏㅁ & 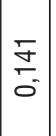 & $\begin{array}{l}\stackrel{m}{m} \\
\bar{c}\end{array}$ & 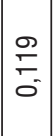 & $\mid \begin{array}{l}0 \\
N \\
0 \\
0\end{array}$ & $\mid \begin{array}{l}\pi \\
0 \\
0\end{array}$ & $\begin{array}{l}\hat{N} \\
\overline{0}\end{array}$ & $\begin{array}{l}0 \\
0 \\
0 \\
0\end{array}$ & $\begin{array}{l}\frac{2}{2} \\
\frac{2}{6} \\
0\end{array}$ & $\mid \begin{array}{l}1 \\
\overline{6} \\
0 \\
0\end{array}$ & $\begin{array}{l}8 \\
0 \\
0\end{array}$ & $\frac{\pi}{0}$ \\
\hline & 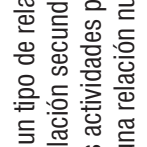 & 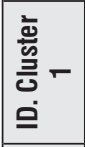 & 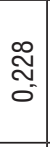 & $\begin{array}{l}0 \\
0 \\
0 \\
0\end{array}$ & $\frac{\infty}{\stackrel{\infty}{\circ}}$ & $\begin{array}{l}\frac{0}{2} \\
\stackrel{\Xi}{0}\end{array}$ & $\frac{\mathscr{P}}{5}$ & $\begin{array}{l}\widetilde{0} \\
\text { m. } \\
0 \\
0\end{array}$ & $\frac{10}{\frac{10}{0}}$ & $\begin{array}{l}\bar{\delta} \\
\overline{0} \\
0\end{array}$ & $\begin{array}{c}\text { శ్ల్ } \\
\text { ర }\end{array}$ & $\mid \begin{array}{l}\frac{1}{2} \\
\frac{1}{0} \\
0\end{array}$ & $\begin{array}{l}\stackrel{2}{\circ} \\
0\end{array}$ & $\left|\begin{array}{l}0 \\
\stackrel{2}{6} \\
0 \\
0\end{array}\right|$ & $\begin{array}{l}\frac{\pi}{\circ} \\
\vdots\end{array}$ & $\mid \begin{array}{l}0 \\
0 \\
0 \\
0\end{array}$ & $\begin{array}{l}\infty \\
\infty \\
0\end{array}$ & $\mid \begin{array}{l}\hat{\sigma} \\
\frac{0}{0}\end{array}$ & $\begin{array}{l}0 \\
f \\
0\end{array}$ & $\mid \begin{array}{c}0 \\
0 \\
0 \\
0 \\
0\end{array}$ & $\begin{array}{l}₫ \\
\vdots \\
0\end{array}$ & $\begin{array}{l}\overline{\text { og }} \\
\text { ô. }\end{array}$ \\
\hline 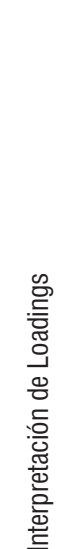 & 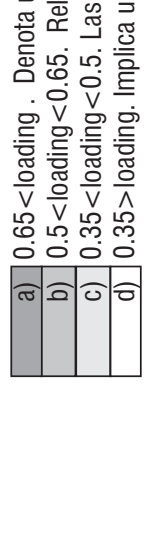 & 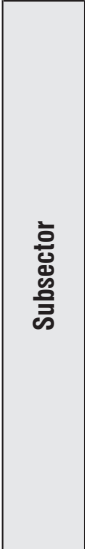 & 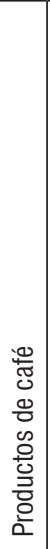 & 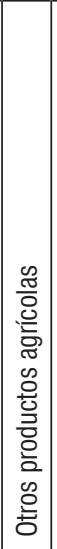 & 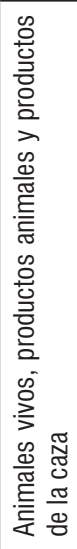 & 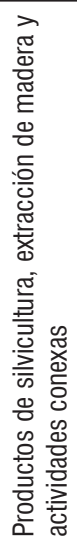 & 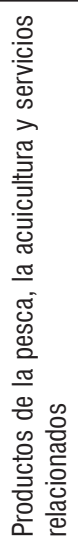 & 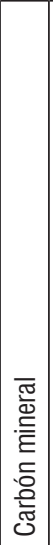 & 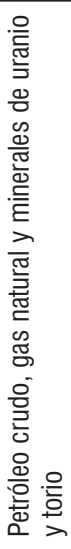 & 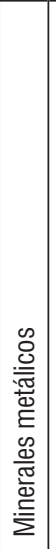 & 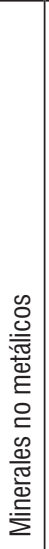 & 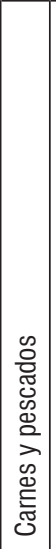 & 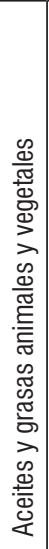 & 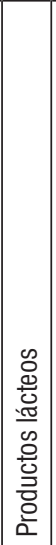 & 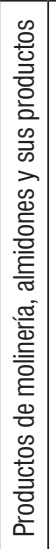 & 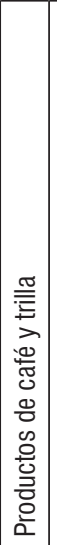 & 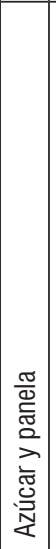 & 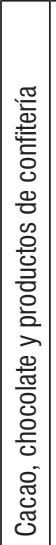 & 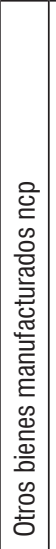 & 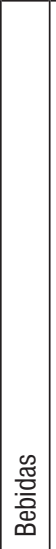 & 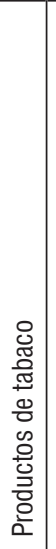 & 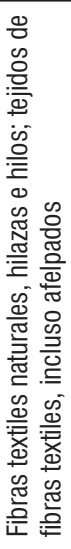 \\
\hline
\end{tabular}




\begin{tabular}{|c|c|c|c|c|c|c|c|c|c|c|c|c|c|c|c|c|c|c|c|c|c|c|c|c|}
\hline 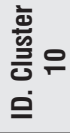 & 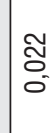 & $\mid \begin{array}{c}\sim \\
\vdots \\
0 \\
0\end{array}$ & శ్ & $\begin{array}{l}\infty \\
\text { O̊ }\end{array}$ & $\begin{array}{l}\mathscr{O} \\
\text { סू }\end{array}$ & $\begin{array}{l}\mathbf{D} \\
0 \\
0\end{array}$ & $\frac{0}{\frac{0}{0}}$ & $\begin{array}{l}\widetilde{\delta} \\
\text { ס्. }\end{array}$ & $\begin{array}{l}0 \\
0 \\
0\end{array}$ & $\begin{array}{l}\infty \\
\stackrel{8}{f} \\
0 \\
0\end{array}$ & $\begin{array}{l}8 \\
0 \\
0\end{array}$ & $\mid \begin{array}{l}\mathscr{T} \\
0 \\
0 \\
0\end{array}$ & $\mid \begin{array}{l}\tau \\
0 \\
0 \\
0\end{array}$ & $\left|\begin{array}{l}0 \\
0 \\
0 \\
0\end{array}\right|$ & $\mid \begin{array}{l}\infty \\
0 \\
0 \\
0\end{array}$ & $\overline{0}$ & $\left|\begin{array}{c}\hat{N} \\
0\end{array}\right|$ & $\begin{array}{l}\stackrel{2}{E} \\
\underbrace{}_{0}\end{array}$ & $\begin{array}{l}\mathfrak{Z} \\
\underset{\mathfrak{Z}}{0}\end{array}$ & $\begin{array}{l}\text { O } \\
\text { : }\end{array}$ & $\begin{array}{l}\stackrel{R}{~} \\
\frac{0}{0}\end{array}$ & $\begin{array}{l}\infty \\
0 \\
0 \\
0\end{array}$ & $\begin{array}{l}\hat{\sigma} \\
0 \\
0\end{array}$ & $\begin{array}{l}0 \\
0 \\
0 \\
0\end{array}$ \\
\hline 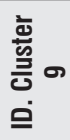 & $\approx$ & $\begin{array}{l}0 \\
0 \\
0\end{array}$ & $\begin{array}{l}\text { Iै } \\
\text { O. }\end{array}$ & $\begin{array}{l}\frac{\sigma}{0} \\
\dot{0} \\
0^{\prime}\end{array}$ & \begin{tabular}{l}
$\mathbb{O}$ \\
\multirow{\sigma}{0}{} \\
0
\end{tabular} & 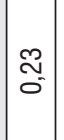 & $\begin{array}{l}\Delta \\
0\end{array}$ & $\frac{\infty}{\frac{m}{6}}$ & $\mid \begin{array}{l}0 \\
0 \\
0 \\
0\end{array}$ & $\begin{array}{l}\widetilde{\sigma} \\
\text { o } \\
0\end{array}$ & $\begin{array}{l}\text { ¿ } \\
0 \\
0\end{array}$ & $\mid \begin{array}{l}\nexists \\
0 \\
0\end{array}$ & $\begin{array}{l}\infty \\
0 \\
0 \\
0\end{array} \mid$ & $\left|\begin{array}{c}\bar{\sigma} \\
0 \\
0\end{array}\right|$ & $\left|\begin{array}{c}0 \\
0 \\
0 \\
0\end{array}\right|$ & $\begin{array}{c}\tilde{m} \\
0 \\
0\end{array}$ & $\mid \begin{array}{l}\mathscr{8} \\
0 \\
0\end{array}$ & $\begin{array}{l}\bar{g} \\
\sigma_{0}^{\circ}\end{array}$ & $\begin{array}{l}\infty \\
\tilde{O} \\
0 \\
0\end{array}$ & $\begin{array}{l}\bar{\delta} \\
0 \\
0\end{array}$ & 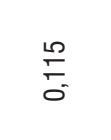 & $\begin{array}{l}\tilde{y} \\
0 \\
0 \\
0\end{array}$ & $\begin{array}{l}\bar{O} \\
\text { Oे }\end{array}$ & $\hat{O}$ \\
\hline 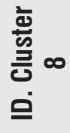 & 芯 & & $\begin{array}{l}\overline{\tilde{B}} \\
\text { o. }\end{array}$ & స్ & $\underset{E}{E}$ & $\begin{array}{l}\mathbf{O} \\
0 \\
0\end{array}$ & $\begin{array}{l}\text { O } \\
\text { O }\end{array}$ & 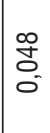 & $\begin{array}{l}g \\
0 \\
0\end{array}$ & 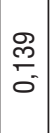 & $\begin{array}{l}\text { : } \\
\text { O. } \\
0\end{array}$ & $\bar{\delta}$ & $\begin{array}{l}0 \\
0 \\
0 \\
0\end{array} \mid$ & $\mid \begin{array}{c}\tilde{O} \\
0 \\
0\end{array}$ & $\left|\begin{array}{l}\infty \\
0 \\
\vdots \\
0\end{array}\right|$ & $\mid \begin{array}{l}0 \\
0 \\
0 \\
0\end{array}$ & $\mid \begin{array}{l}0 \\
0 \\
0\end{array}$ & $\begin{array}{l}\widetilde{o} \\
\text { o. }\end{array}$ & $\begin{array}{l}8 \\
0\end{array}$ & Б) & $\frac{8}{\circ}$ & $\begin{array}{l}\bar{o} \\
0 \\
0\end{array}$ & 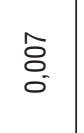 & $\begin{array}{l}\infty \\
\stackrel{0}{0}\end{array}$ \\
\hline 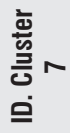 & 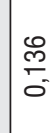 & $\begin{array}{l}\infty \\
0 \\
0\end{array}$ & \begin{tabular}{l}
\multirow{+}{*}{} \\
0 \\
0
\end{tabular} & $\begin{array}{l}\text { o } \\
\text { O. }\end{array}$ & 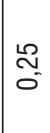 & $\mid \begin{array}{l}0 \\
0 \\
0 \\
0\end{array}$ & $\begin{array}{l}\hat{O} \\
\text { O }\end{array}$ & $\begin{array}{l}\infty \\
0 \\
0 \\
0\end{array}$ & $\mid \begin{array}{c}\widetilde{\Xi} \\
\tilde{O} \\
\tilde{0}\end{array}$ & $\begin{array}{l}\hat{o g} \\
o \\
0\end{array}$ & $\begin{array}{l}\text { đั } \\
\text { - }\end{array}$ & $\mid \begin{array}{l}\infty \\
\vdots \\
0 \\
0\end{array}$ & $\left|\begin{array}{l}2 \\
0 \\
0 \\
0\end{array}\right|$ & $\left|\begin{array}{l}\tilde{\delta} \\
0 \\
0\end{array}\right|$ & $\begin{array}{l}8 \\
0\end{array}$ & $\mid \begin{array}{c}\overline{0} \\
0 \\
0\end{array}$ & $\left|\begin{array}{l}\infty \\
\\
\\
0\end{array}\right|$ & $\begin{array}{l}8 \\
0 \\
0\end{array}$ & $\begin{array}{l}\bar{o} \\
\dot{0} \\
0\end{array}$ & זٓ & $\begin{array}{l}\bar{\delta} \\
0\end{array}$ & $\tilde{\delta}$ & 管 & $\begin{array}{l}\text { đo } \\
: 0 \\
0\end{array}$ \\
\hline 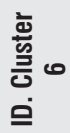 & ơ & $\frac{n}{0}$ & $\begin{array}{l}0 \\
\text { O̊ } \\
0 \\
0\end{array}$ & $\begin{array}{l}\text { \& } \\
\text { O̊ }\end{array}$ & 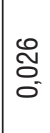 & $\mid \begin{array}{l}\bar{E} \\
\overline{0} \\
0\end{array}$ & $\begin{array}{l}\text { ō } \\
\text { б. }\end{array}$ & $\begin{array}{l}\text { o. } \\
\text { O. }\end{array}$ & $\begin{array}{l}\hat{\tilde{o}} \\
0 \\
0\end{array}$ & $\begin{array}{l}0 \\
0 \\
0 \\
0 \\
0\end{array}$ & $\begin{array}{l}\mathscr{2} \\
\stackrel{0}{0}\end{array}$ & $\left|\begin{array}{l}0 \\
o \\
0 \\
0\end{array}\right|$ & $\begin{array}{l}0 \\
0 \\
0 \\
0\end{array}$ & $\mid \begin{array}{l}\tilde{0} \\
0 \\
0 \\
0\end{array}$ & $\mid \begin{array}{l}\infty \\
0 \\
0\end{array}$ & $\frac{m}{0}$ & $\left|\begin{array}{l}\hat{\sigma} \\
\bar{c}\end{array}\right|$ & 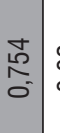 & $\tilde{O}$ & 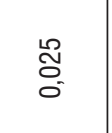 & $\begin{array}{l}\text { I. } \\
\text { O. } \\
0\end{array}$ & \begin{tabular}{l}
\multirow{\Xi}{\Delta}{} \\
0 \\
0
\end{tabular} & $\stackrel{F}{E}$ & ণ̊ \\
\hline 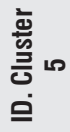 & $\overline{8}$ & \begin{tabular}{|l}
$\overline{\tilde{g}}$ \\
0 \\
0
\end{tabular} & $\begin{array}{l}\mathscr{8} \\
\text { O. }\end{array}$ & 艿 & $\begin{array}{l}0 \\
0 \\
0 \\
0\end{array}$ & $\begin{array}{l}0 \\
0 \\
0 \\
0\end{array}$ & $\begin{array}{l}8 \\
0 \\
0\end{array}$ & 잉 & $\begin{array}{l}8 \\
8 \\
0 \\
0\end{array}$ & 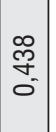 & $\begin{array}{l}\text { Iี } \\
\text { Oे }\end{array}$ & $\mid \begin{array}{c}\mathcal{Z} \\
\tilde{O} \\
0\end{array}$ & $\begin{array}{l}\tilde{O} \\
0 \\
0\end{array}$ & $\left|\begin{array}{c}\mathcal{Z} \\
\tilde{0} \\
0\end{array}\right|$ & $\mid \begin{array}{l}\infty \\
0 \\
0 \\
0\end{array}$ & 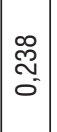 & $\mid$\begin{tabular}{|c|}
$m$ \\
0 \\
0 \\
0
\end{tabular} & $\begin{array}{l}\tilde{Z} \\
\tilde{\delta} \\
0\end{array}$ & $\begin{array}{c}\bar{\delta} \\
0\end{array}$ & శ్ & $\begin{array}{l}\mathscr{8} \\
0 \\
0\end{array}$ & $\stackrel{\simeq}{\stackrel{0}{0}}$ & $\stackrel{\stackrel{\infty}{ \pm}}{\stackrel{0}{0}}$ & $\tilde{O}_{0}$ \\
\hline 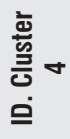 & ס & $\mid \begin{array}{l}\tilde{N} \\
0 \\
0\end{array}$ & 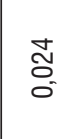 & $\begin{array}{l}\hat{\theta} \\
0\end{array}$ & $\underset{\text { J }}{\text { J }}$ & $\left|\begin{array}{l}\stackrel{2}{2} \\
\vdots \\
0\end{array}\right|$ & $\frac{\infty}{\frac{\infty}{0}}$ & $\begin{array}{l}\mathscr{2} \\
0 \\
0 \\
0\end{array}$ & $\mid \begin{array}{l}\infty \\
0 \\
0 \\
0\end{array}$ & $\begin{array}{l}\text { g } \\
\text { : } \\
0\end{array}$ & $\begin{array}{l}\text { o̊ } \\
\text { o. }\end{array}$ & $\mid \begin{array}{l}\hat{0} \\
0 \\
0\end{array}$ & $\begin{array}{l}\overline{0} \\
0 \\
0\end{array}$ & $\overline{0}$ & $\left|\begin{array}{l}\tilde{N} \\
0 \\
0\end{array}\right|$ & $\begin{array}{l}\underset{N}{0} \\
\vdots\end{array}$ & $\left|\begin{array}{l}0 \\
0 \\
0\end{array}\right|$ & $\begin{array}{c}5 \\
E \\
\bar{O}\end{array}$ & $\begin{array}{l}0 \\
0 \\
0 \\
0\end{array}$ & $\stackrel{8}{8}$ & $\begin{array}{l}\text { : } \\
0 \\
0\end{array}$ & $\begin{array}{l}\widetilde{N} \\
0 \\
0\end{array}$ & $\frac{\cong}{\stackrel{N}{0}}$ & $\begin{array}{l}\text { 邑 } \\
\text { O }\end{array}$ \\
\hline 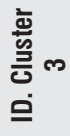 & ్ㅡㅁ & \begin{tabular}{|l} 
\\
0 \\
0 \\
0 \\
0
\end{tabular} & $\begin{array}{l}8 \\
8 \\
0\end{array}$ & $\begin{array}{l}\text { \& } \\
\text { ్ㅗ }\end{array}$ & $\begin{array}{c}\infty \\
\stackrel{\infty}{0} \\
0 \\
0\end{array}$ & 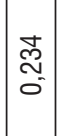 & $\frac{\approx}{\sigma}$ & $\begin{array}{l}\text { L } \\
\text { 只 } \\
0\end{array}$ & $\left|\begin{array}{l}\bar{\delta} \\
\dot{0}\end{array}\right|$ & $\begin{array}{l}\stackrel{2}{0} \\
0 \\
0\end{array}$ & $\frac{\infty}{0}$ & $\mid \begin{array}{c}\bar{m} \\
\overline{0}\end{array}$ & $\begin{array}{l}\bar{N} \\
\bar{O}\end{array}$ & $\mid \begin{array}{l}\infty \\
: \\
0 \\
0\end{array}$ & $\begin{array}{l}\mathscr{0} \\
\mathscr{O} \\
0 \\
0\end{array}$ & 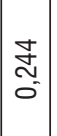 & $\begin{array}{l}\infty \\
0 \\
0\end{array}$ & \begin{tabular}{l|}
10 \\
0 \\
0 \\
0
\end{tabular} & $\begin{array}{l}\stackrel{2}{2} \\
0 \\
0\end{array}$ & $\stackrel{\circ}{\circ}$ & $\begin{array}{l}\tilde{O} \\
\text { Oे }\end{array}$ & 总 & $\begin{array}{l}0 \\
\text { \% } \\
0\end{array}$ & $\begin{array}{l}\text { No } \\
\text { Oे. }\end{array}$ \\
\hline 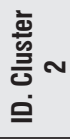 & శ్ & $\begin{array}{l}\text { OD } \\
\overline{0} \\
0\end{array}$ & $\begin{array}{l}\text { Õ } \\
0 \\
0\end{array}$ & 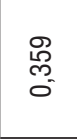 & $\begin{array}{l}\text { \& } \\
\text { : }\end{array}$ & 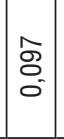 & $\frac{\mathscr{m}}{\sigma_{0}^{\prime}}$ & \& & $\mid \begin{array}{l}\tilde{2} \\
\tilde{O} \\
0\end{array}$ & $\mid \begin{array}{c}\tilde{J} \\
\text { J } \\
0\end{array}$ & $\begin{array}{l}\bar{o} \\
\infty \\
0 \\
0\end{array}$ & $\mid \begin{array}{l}\mathscr{2} \\
\widetilde{d} \\
0\end{array}$ & $\left|\begin{array}{l}\bar{\sigma} \\
\tilde{\sigma}\end{array}\right|$ & $\begin{array}{l}\hat{O} \\
0 \\
0\end{array}$ & 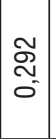 & $\left|\begin{array}{l}\tilde{N} \\
\tilde{o} \\
\tilde{o}\end{array}\right|$ & $\mid \begin{array}{l}\hat{o} \\
0 \\
0\end{array}$ & $\begin{array}{l}\hat{o} \\
0 \\
0\end{array}$ & 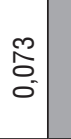 & $\begin{array}{l}\mathscr{0} \\
\infty \\
0 \\
0\end{array}$ & $\begin{array}{l}\text { J } \\
\text { o }\end{array}$ & $\begin{array}{l}\mathscr{0} \\
0 \\
0\end{array}$ & $\tilde{\sigma}$ & $\begin{array}{l}0 \\
\\
0 \\
0\end{array}$ \\
\hline 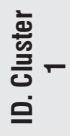 & 䒠 & $\mid \begin{array}{l}\tilde{y} \\
0 \\
0 \\
0\end{array}$ & 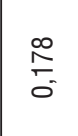 & $\begin{array}{l}\text { 旾 } \\
0 \\
0\end{array}$ & $\begin{array}{c}\mathscr{0} \\
\dddot{m} \\
0 \\
0\end{array}$ & $\begin{array}{l}\mathscr{D} \\
\hat{0} \\
0\end{array}$ & $\frac{8}{\circ}$ & $\begin{array}{l}\text { Oू } \\
\text { O. }\end{array}$ & $\mid \begin{array}{l}0 \\
\delta \\
0 \\
0\end{array}$ & \begin{tabular}{|c}
$\tilde{D}$ \\
0 \\
0 \\
0
\end{tabular} & $\frac{\stackrel{2}{N}}{0}$ & $\begin{array}{l}\widetilde{\Xi} \\
\tilde{O} \\
0\end{array}$ & 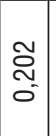 & $\left|\begin{array}{l}\Re \\
\stackrel{2}{0} \\
0\end{array}\right|$ & $\begin{array}{l}\tilde{0} \\
0 \\
0\end{array}$ & $\left|\begin{array}{l}\hat{m} \\
\frac{0}{0}\end{array}\right|$ & $\begin{array}{c}n \\
0 \\
0 \\
0\end{array}$ & $\begin{array}{l}\hat{\circ} \\
0 \\
0 \\
0\end{array}$ & \begin{tabular}{l}
0 \\
\multirow{\sigma}{\sigma}{} \\
0
\end{tabular} & $\begin{array}{l}\text { R } \\
\stackrel{6}{0} \\
0\end{array}$ & $\begin{array}{l}\text { ळ. } \\
\text { ల్ } \\
0\end{array}$ & $\begin{array}{l}\hat{\mathscr{\theta}} \\
0 \\
0\end{array}$ & \begin{tabular}{l}
$\stackrel{2}{2}$ \\
\hdashline \\
0
\end{tabular} & O্: \\
\hline 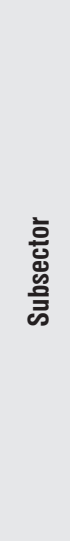 & 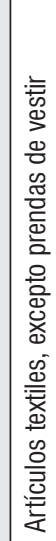 & 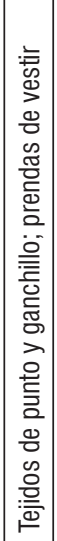 & 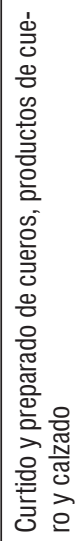 & 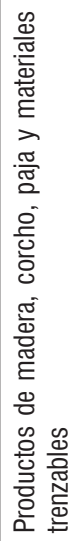 & 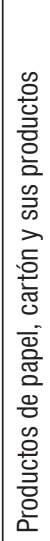 & 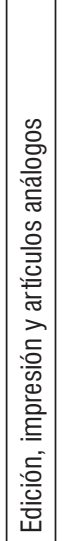 & 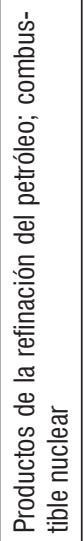 & 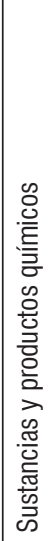 & 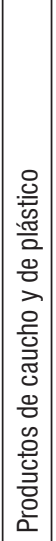 & 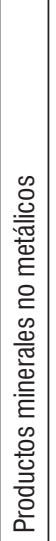 & 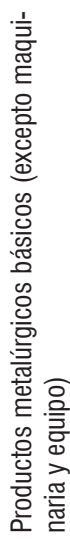 & 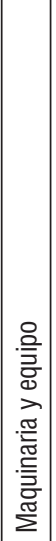 & 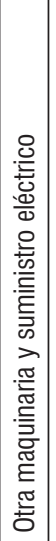 & 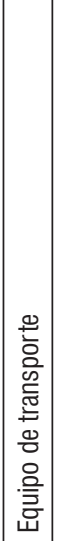 & 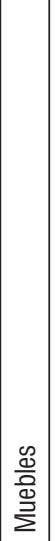 & 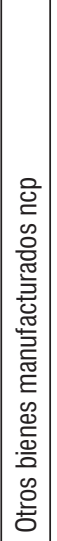 & 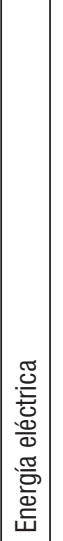 & 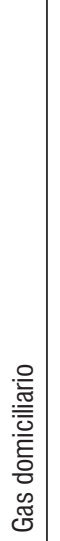 & 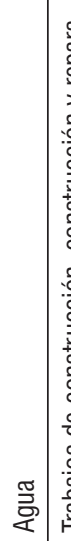 & 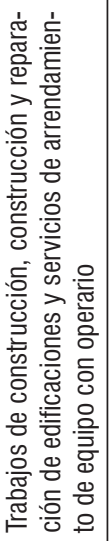 & 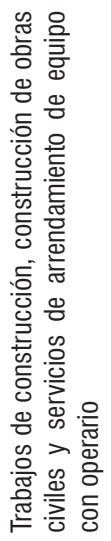 & 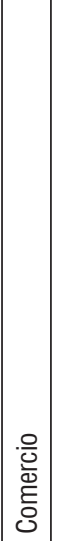 & 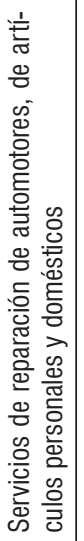 & 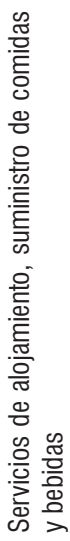 \\
\hline
\end{tabular}




\begin{tabular}{|c|c|c|c|c|c|c|c|c|c|c|c|c|c|c|c|}
\hline 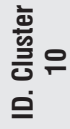 & $\begin{array}{l}8 \\
\delta \\
0\end{array}$ & $\frac{\Xi}{\check{\Xi}}$ & $\begin{array}{l}0 \\
0 \\
0 \\
0\end{array}$ & $\frac{\widetilde{\sigma}}{0}$ & $\begin{array}{l}\overline{8} \\
0 \\
0\end{array}$ & $\begin{array}{l}\hat{\circ} \\
\text { Oे }\end{array}$ & $\frac{10}{=}$ & $\begin{array}{l}\text { Zे } \\
\text { Oे } \\
0\end{array}$ & $\frac{g}{\frac{g}{0}}$ & 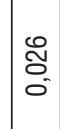 & $\begin{array}{l}n \\
E \\
0\end{array}$ & $\mid \begin{array}{l}\tilde{\mathbb{N}} \\
0 \\
0\end{array}$ & 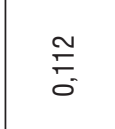 & $\begin{array}{l}\text { 용 } \\
0\end{array}$ & $\begin{array}{l}\widetilde{\delta} \\
\text { Oे } \\
\text { - }\end{array}$ \\
\hline 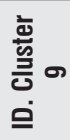 & ${ }_{\infty}^{\infty}$ & 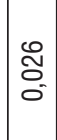 & $\begin{array}{l}\stackrel{L}{\mathbb{Z}} \\
0 \\
0\end{array}$ & $\frac{m}{0}$ & $\frac{N}{\tilde{O}}$ & 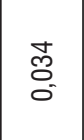 & 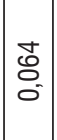 & $\begin{array}{l}\text { षे } \\
\text { O. }\end{array}$ & $\begin{array}{l}\hat{o} \\
\stackrel{0}{0}\end{array}$ & $\bar{\sigma}$ & $\begin{array}{l}8 \\
0 \\
0\end{array}$ & $\mid \begin{array}{l}m \\
m \\
0 \\
0\end{array}$ & $\begin{array}{l}\stackrel{\Xi}{\Xi} \\
\text { O. }\end{array}$ & $\begin{array}{l}\mathscr{D}_{0} \\
\text { O. }\end{array}$ & $\bar{\sigma}$ \\
\hline 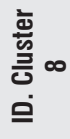 & 宂 & $\begin{array}{l}\bar{\phi} \\
\overline{0}\end{array}$ & \begin{tabular}{|l}
$\overline{\tilde{\sigma}}$ \\
$\overline{0}$
\end{tabular} & $\begin{array}{l}0 \\
0 \\
0 \\
0\end{array}$ & Oo & $\begin{array}{l}\text { \% } \\
\text { O. }\end{array}$ & $\begin{array}{l}\stackrel{2}{0} \\
0\end{array}$ & $\begin{array}{l}\infty \\
\tilde{O} \\
0\end{array}$ & $\begin{array}{l}8 \\
\varnothing \\
0\end{array}$ & 正 & $\begin{array}{l}0 \\
0 \\
0 \\
0\end{array}$ & 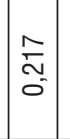 & $\begin{array}{l}\hat{o} \\
\text { o. } \\
0\end{array}$ & O̊. & $\begin{array}{l}\text { o } \\
\varnothing \\
0\end{array}$ \\
\hline 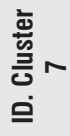 & 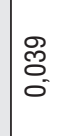 & 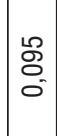 & : & $\begin{array}{l}\dot{8} \\
0 \\
0\end{array}$ & $\begin{array}{l}\text { Z } \\
\text { O }\end{array}$ & 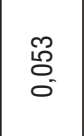 & $\begin{array}{l}\stackrel{2}{0} \\
0\end{array}$ & $\begin{array}{l}0 \\
0 \\
0 \\
0\end{array}$ & $\begin{array}{l}\text { \& } \\
0 \\
0\end{array}$ & $\tilde{\delta}$ & $\mid \begin{array}{l}1 \\
0 \\
0 \\
0\end{array}$ & $\cong$ & $\overline{8}$ & זั. & $\begin{array}{l}0 \\
0 \\
0 \\
0\end{array}$ \\
\hline 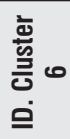 & \& & $\begin{array}{l}\tilde{\Xi} \\
\text { ¿ } \\
0\end{array}$ & $\begin{array}{l}\mathbb{J} \\
\mathbf{O} \\
0\end{array}$ & $\begin{array}{l}\text { స్ } \\
\text { O. }\end{array}$ & $\begin{array}{l}m \\
\vdots \\
0 \\
0\end{array}$ & $\begin{array}{l}\text { 옹 } \\
0\end{array}$ & 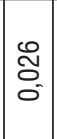 & 용 & $\begin{array}{l}\infty \\
\stackrel{0}{0} \\
0\end{array}$ & $\begin{array}{l}\vec{\sigma} \\
0 \\
0\end{array}$ & $\mid \begin{array}{l}\pi \\
0 \\
0\end{array}$ & $\mid \begin{array}{l}\mathfrak{Y} \\
\tilde{\sigma}\end{array}$ & $\begin{array}{l}\text { \& } \\
\text { Oे }\end{array}$ & চ̄ & శ్లి \\
\hline 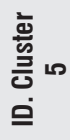 & $\begin{array}{l}\text { \& } \\
\delta \\
0 \\
0\end{array}$ & $\begin{array}{l}1 \\
0 \\
0 \\
0 \\
0\end{array}$ & $\begin{array}{l}\text { ס } \\
\delta \\
0 \\
0\end{array}$ & $\frac{\mathbb{R}}{0}$ & 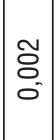 & $\begin{array}{l}\text { o̊ } \\
\text { ర్ }\end{array}$ & $\begin{array}{l}\text { fै } \\
\text { : }\end{array}$ & $\frac{n}{0}$ & $\begin{array}{l}\stackrel{\mathscr{D}}{心} \\
\stackrel{0}{0}\end{array}$ & 0 & $\mid \begin{array}{l}0 \\
0 \\
0\end{array}$ & $\begin{array}{l}\bar{\sigma} \\
0\end{array}$ & 落 & $\begin{array}{l}\mathbb{\Delta} \\
\text { ¿. }\end{array}$ & סू \\
\hline 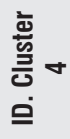 & $\frac{m}{c}$ & $\begin{array}{l}\frac{\sigma}{\sigma} \\
\bar{\sigma}\end{array}$ & 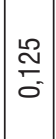 & $\begin{array}{l}\infty \\
\ddot{0} \\
0\end{array}$ & $\begin{array}{l}\Delta \\
0 \\
0\end{array}$ & \begin{tabular}{l}
0 \\
\multirow{O}{0}{} \\
0
\end{tabular} & $\begin{array}{l}8 \\
8 \\
0\end{array}$ & $\stackrel{\Delta}{0}$ & $\begin{array}{l}\stackrel{\circ}{\circ} \\
\frac{0}{0}\end{array}$ & $\begin{array}{l}\tilde{D} \\
0 \\
0 \\
0\end{array}$ & $\mid \begin{array}{l}0 \\
0 \\
0\end{array}$ & $\begin{array}{l}\bar{m} \\
\bar{c}\end{array}$ & $\begin{array}{l}\stackrel{2}{0} \\
\text { O. }\end{array}$ & $\dot{\sigma}_{0}^{+}$ & 0 \\
\hline 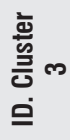 & $\frac{\infty}{N}$ & $\begin{array}{l}0 \\
10 \\
0\end{array}$ & $\frac{\mathfrak{F}}{\tilde{\sigma}}$ & $\begin{array}{l}\text { O্ণ } \\
\text { O }\end{array}$ & $\begin{array}{l}\infty \\
\text { Oo } \\
0 \\
0\end{array}$ & $\begin{array}{l}0 \\
\stackrel{0}{0} \\
0\end{array}$ & $\begin{array}{l}\mathscr{\delta} \\
0 \\
0\end{array}$ & $\overline{0}$ & $\frac{g}{\frac{g}{0}}$ & $\begin{array}{c}0 \\
\vdots \\
0\end{array}$ & $\begin{array}{l}\hat{\theta} \\
0 \\
0\end{array}$ & $\begin{array}{l}\frac{1}{8} \\
0 \\
0\end{array}$ & 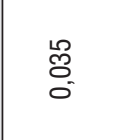 & ஜூ: & $\begin{array}{l}\frac{10}{0} \\
0 \\
0\end{array}$ \\
\hline 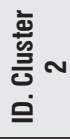 & ஜ & $\begin{array}{l}\widetilde{\delta} \\
\text { ठ. } \\
0\end{array}$ & $\begin{array}{l}\hat{\theta} \\
0 \\
0\end{array}$ & $\begin{array}{l}\hat{o} \\
\text { o. }\end{array}$ & 要 & $\begin{array}{l}\text { ஜ } \\
\text { O } \\
0\end{array}$ & $\begin{array}{l}8 \\
8 \\
0\end{array}$ & $\begin{array}{l}\text { 芯 } \\
\text { O. }\end{array}$ & $\begin{array}{l}\text { 号 } \\
0 \\
0\end{array}$ & $\begin{array}{l}\tilde{R} \\
\stackrel{0}{0} \\
0\end{array}$ & $\left|\begin{array}{l}\infty \\
\delta \\
0 \\
0\end{array}\right|$ & 莕 & $\begin{array}{l}\widetilde{O} \\
\text { ¿ }\end{array}$ & 兽 & $\begin{array}{l}8 \\
0 \\
0\end{array}$ \\
\hline 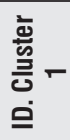 & 苋 & 离 & $\begin{array}{l}m \\
0 \\
0\end{array}$ & $\begin{array}{l}\mathscr{8} \\
\stackrel{8}{0} \\
0\end{array}$ & $\begin{array}{l}\hat{N} \\
\sigma \\
\sigma\end{array}$ & $\begin{array}{l}\text { ஜू } \\
\text { : }\end{array}$ & $\begin{array}{l}\mathscr{0} \\
: \\
0 \\
0\end{array}$ & $\begin{array}{l}\text { g. } \\
\hat{\sigma} \\
0\end{array}$ & ஜ్ల & $\begin{array}{l}2 \\
: \\
O \\
0 \\
0\end{array}$ & $\left|\begin{array}{c}8 \\
\infty \\
\infty \\
0\end{array}\right|$ & 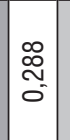 & $\begin{array}{l}\text { L̊ } \\
\text { \&్ } \\
0\end{array}$ & $\begin{array}{l}\mathscr{\Omega} \\
0 \\
0\end{array}$ & $\begin{array}{l}\mathbb{R} \\
o \\
0 \\
0\end{array}$ \\
\hline 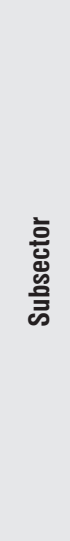 & 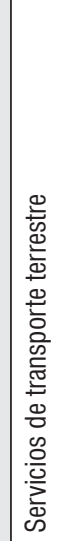 & 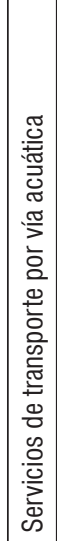 & 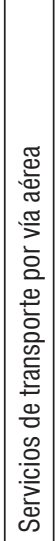 & 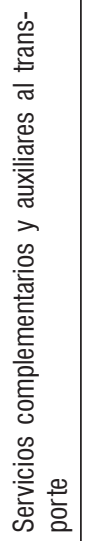 & 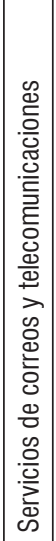 & 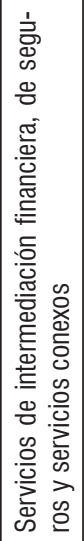 & 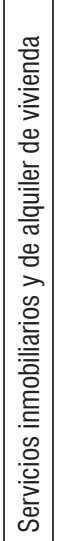 & 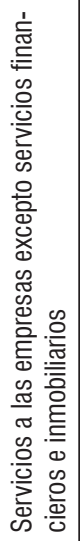 & 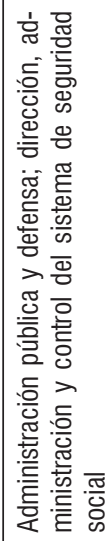 & 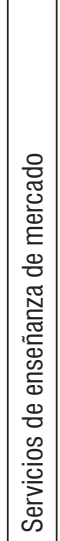 & 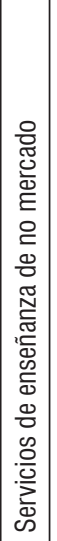 & 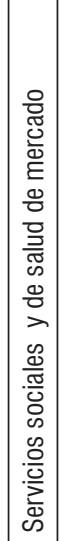 & 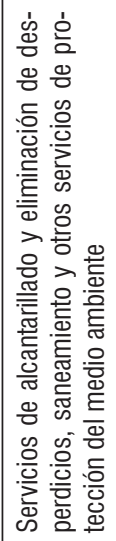 & 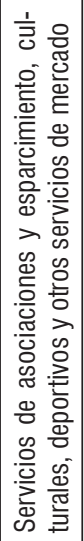 & 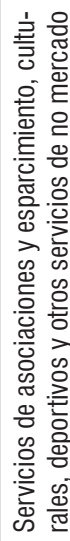 \\
\hline
\end{tabular}

